\title{
Strain effects on silicon donor exchange: Quantum computer architecture considerations
}

\author{
Belita Koiller ${ }^{1,2}$, Xuedong $\mathrm{Hu}^{1}$, and S. Das Sarma ${ }^{1}$ \\ ${ }^{1}$ Department of Physics, University of Maryland, College Park, MD 20742-4111 \\ 2 Instituto de Física, Universidade Federal do Rio de Janeiro, 21945, Rio de Janeiro, Brazil
}

(March 17, 2021)

\begin{abstract}
Proposed Silicon-based quantum computer architectures have attracted attention because of their promise for scalability and their potential for synergetically utilizing the available resources associated with the existing infrastructure of the powerful Si technology. Quantitative understanding of and precise physical control over donor (e.g. Phosphorus) exchange are crucial elements in the physics underlying the proposed Si-based quantum computer hardware. An important potential problem in this context is that inter-valley interference originating from the degeneracy in the $\mathrm{Si}$ conduction band edge causes fast oscillations in donor exchange coupling, which imposes significant constraints on the Si quantum computer architecture. In this paper we consider the effect of external strain on Si donor exchange in the context of quantum computer hardware. We study donor electron exchange in uniaxially strained $\mathrm{Si}$, since strain partially lifts the valley degeneracy in the bulk. In particular, we focus on the effects of donor displacements among lattice sites on the exchange coupling, investigating whether inter-valley interference poses less of a problem to exchange coupling of donors in strained Si. We show, using the Kohn-Luttinger envelope function approach, that fast oscillations in exchange coupling indeed disappear for donor pairs that satisfy certain conditions for their relative positions, while in other situations the donor exchange coupling remains oscillatory, with periods close to interatomic spacing. We also comment on the possible role of controlled external strain in the design and fabrication of Si quantum computer architecture.
\end{abstract}

PACS numbers: 71.55.Cn, 03.67.Lx, 85.30.Vw

\section{INTRODUCTION}

Advances in quantum computing software research, 1 such as the invention of factorization algorithm and quantum error correction codes, have prompted active and extensive search for an appropriate physical system for implementation of quantum computation.2 For example, there has been considerable recent interest in the study of donor impurities in silicon, particularly the $\mathrm{Si}^{31} \mathrm{P}$ system, because of the potential of the monovalent ${ }^{31} \mathrm{P}$ impurities to act as fundamental units of a solid state quantum computer (QC). The first Si-based QC architecture, proposed by Kane, has drawn immense interest from the experimental community. In Kane's proposal the nuclear spins of phosphorus $\left({ }^{31} \mathrm{P}\right)$ donors are the quantum bits (qubits), while the donor electrons act as shuttles between neighboring nuclear spins. Isotopically purified ${ }^{28} \mathrm{Si}$ host provides a quiet environment with very long coherence times for both donor electronic and nuclear spins 4 since it has been known for a long time 6 that the electron spin coherence time in $\mathrm{Si}$ is extremely long. Two-qubit operations, which are required for a universal quantum computer, involve precise control over electron-efectron exchanged and electron-nucleus hyperfine interactions. A closely related alternative design was proposed later, involving ${ }^{31} \mathrm{P}$ donors in $\mathrm{Si} / \mathrm{Ge}$ heterostructures, with the spins of the donor electrons (rather than the nuclear spins) serving as qubits. The basic ingredients underlying the Si QC proposals are schematically presented and described in Fig. 1. There are also other recent QC proposals based on Si or Si/Ge host materials. 89 Although these are interesting and promising proposals, particularly due to the extensive infrastructural base available in the existing Si microelectronics technology, there are formidable experimental challenges to be overcome in device fabrication, coherent control, system integration, and single spin measurement, among other important issues, before a Si-based QC architecture could become an experimental reality even on a laboratory scale.

Recently, there has been significant progress in the fabrication of donor arrays in silicon single crystal, using both "top-down" approach with ion-implantation, and a "bottom-up" approach with MBE growth and STM technique.10 Theoretically, we have recently shown 11 that electron exchange in bulk silicon has fast atomic scale spatial oscillations due to the valley interference arising from the very special six-fold degeneracy of the bulk Si conduction band. These oscillations place heavy burdens on the device fabrication and coherent control because of the extremely high precision requirement for plafing each donor inside the Si unit cell and/or for controlling the external gate voltages.

Several authorst.5 have pointed out that Si-based quantum computing architectures involving epitaxial $\mathrm{Si}_{1} / \mathrm{Si}_{1-x} \mathrm{Ge}_{x}$ heterostructures possess some practical advantages over the original scheme based on relaxed bulk Si.3 One of the motivations is the need for a barrier material separating the Si host containing ${ }^{31} \mathrm{P}$ donors from the conducting gates 
(see Fig 1). The Si/barrier-material interface must be free of structural defects. Although high quality Si/oxide barriers can be fabricated, $\mathrm{Si} / \mathrm{Si}_{1-x} \mathrm{Ge}_{x}$ and $\mathrm{Si} / \mathrm{Si}_{1-x} \mathrm{C}_{x}$ are strong candidates for $\mathrm{QC}$ architectures because of the good interface quality associated with their epitaxial nature. These alloys have advantages over the oxide barriers (e.g. $\mathrm{SiO}_{2}$ ) in terms of chemical compatibility among the group IV elements, which share the diamond lattice structure, while still providing sufficiently high barriers for the donor electrons to prevent leak into/from the gates. In spite of sharing the same lattice structure, $\mathrm{C}, \mathrm{Si}$ and Ge present very large lattice mismatches (lattice parameters $3.57 \AA$, 5.43 $\AA$, and $5.66 \AA$, respectively), leading to the presence of strained layers in heterostructures containing commensurately grown materials. The highly strained nature of these epitaxial systems (arising from their large lattice mismatch) in fact is crucial in producing high quality defect free interface since dislocations are avoided due to the strain build-up.

Given our earlier finding of band-degeneracy-induced strong oscillations in the donor exchange energy (as a function of individual donor positioning within the Si unit cell) of bulk $\mathrm{Si}$, it is natural to ask how donor exchange behaves in strained Si heterostructure or quantum well systems, where one must now incorporate strain effects in the exchange calculation. In this paper we carry out such a calculation of strain effects on exchange energy, and emphasize an additional feature of ${ }^{31} \mathrm{P}$-doped strained Si quantum wells (QWs) favoring such architectures over architectures based on relaxed bulk Si for QC implementation. Recall that two-qubit operations of a Si QC rely on the exchange coupling between the wave functions of neighboring donor atoms. The six-fold degeneracy in the conduction band edge of $\mathrm{Si}$ leads to oscillations in the exchange coupling 11 which pose formidable experimental problems in controlling both the fabrication (in terms of donor positioning within the crystal) and the actual operation (in terms of surface gate bias) of the QC. We demonstrate below that these problems are partially solved for impurity pairs in strained Si grown over $\mathrm{Si} / \mathrm{Ge}$ alloys due to altered lattice and band structure. Unfortunately, however, we find that some parts of the exchange oscillation problem persist even in these strained systems as we discuss in this paper.

In the following, we first discuss how to describe theoretically the effects of strain in silicon. We then discuss the calculation of the donor exchange energy via an effective Heitler-London approximation. Finally we present our results and discuss their implications and validity. The details of our Heitler-London calculation are given in the Appendix.

\section{THEORETICAL APPROACH}

\section{A. Strain in Si layers}

Epitaxial growth techniques such as molecular beam epitaxy (MBE) allow the fabrication of lattice-mismatched heterostructures free of misfit defect generation when the layers are sufficiently thin (and the lattice mismatch is not extremely large) 12 The mismatch is completely accommodated by uniform lattice strain and the interatomic distances parallel to the interfacial plane, i.e. the "effective" lattice constants in the plane, remain equal to the equilibrium value of the substrate material. The lattice constants perpendicular to the interface adjust appropriately in order to minimize the total elastic energy in the strained layer. This so-called commensurate growth lowers the energy of the interfacial atoms at the expense of increasing the elastic (strain) energy stored in the chemical bonds in the coherently strained layers.13 We refer to these layers as uniaxially strained. Depending on whether the interatomic distances parallel to the interface are larger or smaller than the relaxed equilibrium value, the strain is called tensile or compressive. The structural variations involved in this process modify the electronic properties of the layered materials and consequently the substitutional impurities in the strained layers.

Here let us consider the important example of $\mathrm{Si}$ grown over a relaxed $\mathrm{Si}_{1-x} \mathrm{Ge}_{x}$ substrate on a (001) interface. Successful commensurate growth of such heterostructures has been previously reported. 14.15 The average alloy lattice parameter, which follows Vegard's law very closely, 16 defines the Si layer lattice parameter parallel to the interface: $\mathrm{a}_{\|}=(1-x) \mathrm{a}_{\mathrm{Si}}+x \mathrm{a}_{\mathrm{Ge}}$. The lattice parameter perpendicular to the (001) interface is also modified in the strained $\mathrm{Si}$ layer with respect to its equilibrium value. According to macroscopic elasticity theory, $\mathrm{a}_{\perp}=\mathrm{a}_{\mathrm{Si}}+2\left(c_{12} / c_{11}\right)\left(\mathrm{a}_{\mathrm{Si}}\right.$ - aw. Here $c_{12}=16.577 \mathrm{dyn} / \mathrm{cm}^{2}$ and $c_{11}=6.393 \mathrm{dyn} / \mathrm{cm}^{2}$ are elastic constants with values given for relaxed bulk Si.17 The elasticity theory predictions for the crystal lattice structures in Si/Ge heterostructures were confirmed by $a b$ initio calculations by Van de Walle and Martin. 13 For an alloy with $x=0.2$, the above relations yield a $\|=5.474$ $\AA$ and $\mathrm{a}_{\perp}=5.396 \AA$, i.e., distortions of less than $1 \%(+0.8 \%$ and $-0.6 \%)$ with respect to the equilibrium value of $\mathrm{a}_{\mathrm{Si}}=5.43 \AA$.

Uniaxially strained $\mathrm{Si}$ has lower symmetry than relaxed $\mathrm{Si}$, and thus a different reciprocal lattice (body centered tetragonal instead of body centered cubic) and Brillouin zone (BZ). Herring and Vogt 18 have shown that the small tetragonal distortions cause changes in the conduction band minima of Si that can be quantitatively described by shifts in the energies of the local minima of the six valleys, while the reciprocal-space positions and shapes of the constant-energy surfaces remain unchanged to first order in strain. The energy shifts are given in terms of the amount of distortion and of the uniaxial and dilation deformation potentials, $\Xi_{u}$ and $\Xi_{d}$, respectively. For Si grown over 
relaxed $\mathrm{Si}_{1-x} \mathrm{Ge}_{x}$ (001) substrate, $\Xi_{d}$ leads to uniform shifts of the six valley minima. Therefore, as discussed below, only $\Xi_{u}$ is relevant for our study of ${ }^{31} \mathrm{P}$ donor wave functions.

\section{B. Effective Hamiltonian for a donor electron in strained $\mathrm{Si}$}

The conduction band edge of relaxed bulk Si consists of six degenerate minima located along the $\langle 100\rangle$ directions, at about $85 \%$ away from the BZ center, towards the zone boundary at X points. Within the Kohn-Luttinger envelope function approach 19 the ground state for a donor electron in $\mathrm{Si}$ is also six-fold degenerate, and the corresponding states are labeled by the reciprocal-space points $\mathbf{k}_{\mu}$ at which the valley minima occur. For definiteness we follow the sequence: $\mu=1,2, \ldots, 6 \leftrightarrow z,-z, y,-y, x,-x$, with the envelope functions given by (e.g. for $\mu= \pm z) \underline{Q}$

$$
F_{ \pm z}(\mathbf{r})=\frac{1}{\sqrt{\pi a^{2} b}} e^{-\left[\left(x^{2}+y^{2}\right) / a^{2}+z^{2} / b^{2}\right]^{1 / 2}} .
$$

The effective Bohr radii for Si from a variational calculation are $a=25.09 \AA$ and $b=14.43 \AA .1$

A substitutional impurity breaks the translatipmal symmetry of the host crystal, leading to intervalley scattering effects known as the valley-orbit interactions, 20 , 21 which split the unperturbed six-fold degenerate donor electron ground state into a singlet $\left(A_{1}\right)$, a triplet $\left(T_{1}\right)$ and a doublet $(E)$, with the ground state being a non-degenerate state of $A_{1}$ symmetry. We follow the perturbative treatment of Baldereschi, ${ }^{2}$ which starts from the six degenerate ground-state functions calculated within the one-valley Kohn-Luttinger variational approach. The low energy donor electron wave functions can be expanded in the basis spanned by the six valleys

$$
\psi(\mathbf{r})=\sum_{\mu=1}^{6} \alpha_{\mu} F_{\mu}(\mathbf{r}) \phi_{\mu}(\mathbf{r}),
$$

where $\phi_{\mu}(\mathbf{r})=u_{\mu}(\mathbf{r}) e^{i \mathbf{k}_{\mu} \cdot \mathbf{r}}$ are the pertinent Bloch wave functions and $\sum_{\mu=1}^{6}\left|\alpha_{\mu}\right|^{2}=1$. Each $\alpha_{\mu}$ coefficient is in general referred to as $\mu$-valley population, while a set of 6 coefficients defines the so-called valley composition of a donor electron state.

To determine the donor electron wave function, we include as perturbations two types of intervalley coupling, for valleys on perpendicular symmetry directions (e.g. $x, z$ ) and for those on opposite symmetry directions (e.g. $z$, $-z)$. We represent these couplings by $-\Delta_{C}$ and $-\Delta_{C}(1+\delta)$, respectively. The experimental values 22 for the relative splittings among the $A_{1}, T_{1}$ and $E$ levels for ${ }^{31} \mathrm{P}$ donors in $\mathrm{Si}$ are obtained as $\Delta_{C}=2.16 \mathrm{meV}$ and $\delta=-0.3$. Taking valley-orbit scattering into consideration through these parameters leads to a binding energy of $40 \mathrm{meV}$ for the $A_{1}$ ground state, in quite a good agreement with the experimental value of $45.5 \mathrm{meV} 22$ More accurate estimates of the binding energy were obtained through non-perturbative variational treatments, 21 which however rely on a spherical-band approximation and adopt s-like hydrogenic trial envelope functions. Since our goal here is to obtain a realistic description of the ground state wave function for the donor electron rather than the precise values of the binding energy, we employ the perturbative approach with anisotropic envelope functions. For our considerations of $\mathrm{Si}$ QC hardware architecture, which focus on electron exchange due to wavefunction overlap, the perturbative envelope function approximation that we employ is quite adequate.

Uniaxial strain along [001] induces different valley shifts for the two local minima along the $z$ axis $(\mu=1,2)$ compared to the other four along $x$ and $y$ axis $(\mu=3,4,5,6)$. Within the subspace spanned by the electron wave functions at the six conduction band minima $\left\{F_{\mu}(\mathbf{r}) \phi_{\mu}(\mathbf{r})\right\}_{\mu=1,6}$, the donor ground and lower excited states may be conveniently obtained as the eigenvectors of the effective perturbation Hamiltonian in the form of a $6 \times 6$ matrix which, for ${ }^{31} \mathrm{P}$ donors in a $\mathrm{Si} \mathrm{QW}$ uniaxially strained along the $z$ axis, is written a ${ }^{23}$

$$
H=H_{v o}+H_{\text {strain }}+H_{\mathrm{z}}=-\Delta_{C}\left(\begin{array}{cccccc}
0 & 1+\delta & 1 & 1 & 1 & 1 \\
1+\delta & 0 & 1 & 1 & 1 & 1 \\
1 & 1 & 0 & 0+\delta & 1 & 1 \\
1 & 1 & 1+\delta & 0 & 1 & 1 \\
1 & 1 & 1 & 1 & 0 & 1+\delta \\
1 & 1 & 1 & 1 & 1+\delta & 0
\end{array}\right)
$$




$$
+\Delta_{C}\left(\begin{array}{cccccc}
2 \chi & 0 & 0 & 0 & 0 & 0 \\
0 & 2 \chi & 0 & 0 & 0 & 0 \\
0 & 0 & -\chi & 0 & 0 & 0 \\
0 & 0 & 0 & -\chi & 0 & 0 \\
0 & 0 & 0 & 0 & -\chi & 0 \\
0 & 0 & 0 & 0 & 0 & -\chi
\end{array}\right)+\left(\begin{array}{cccc}
-\Delta & \Omega & 0 & \cdots \\
\Omega^{*} & -\Delta & 0 & \\
0 & 0 & 0 & \\
\vdots & & & \ddots \\
& & &
\end{array}\right) .
$$

Note that the order of the rows and columns of the perturbation Hamiltonian $H$ follows the sequence in $\mu$ given above Eq. (11). Furthermore, since our concern here is the donor wave functions, any shift in $H$ proportional to the identity has been neglected, so that the eigenvalues of $H$ give the correct relative splittings among the eigenstates, but not the binding energies. The first term of the perturbation Hamiltonian, $H_{v o}$, gives the valley-orbit scattering due to the presence of ${ }^{31} \mathrm{P}$ donors in unstrained $\mathrm{Si}$ discussed above 20 The second term, $H_{\text {strain }}$, introduces the relative energy shifts due to uniaxial strain along [001] in terms of a dimensionless scalar valley strain parameter $\chi$ defined in terms of strain parameters and the valley-orbit scattering matrix element 23

$$
\chi=\frac{\Xi_{u} e_{T}}{3 \Delta_{C}} .
$$

Here the uniaxial strain parameter $\Xi_{u} 18$ is approximately $8.6 \mathrm{eV}$ for the Si conduction band edge, 24,25 while $e_{T}=e_{\mathrm{zz}}-$ $e_{\mathrm{xx}}$ is the difference between the diagonal components of the strain tensor for uniaxial strain along $z: e_{\mathrm{zz}}=\mathrm{a}_{\perp} / \mathrm{a}_{\mathrm{Si}}-1$ and $e_{\mathrm{xx}}=\mathrm{a}_{\|} / \mathrm{a}_{\mathrm{Si}}-1$, so that the valley strain parameter $\chi$ is linearly related to the $\mathrm{Si}_{1-x} \mathrm{Ge}_{x}$ alloy composition $x$ as

$$
\chi=\frac{\Xi_{u}}{3 \Delta_{C}} \frac{\mathrm{a}_{\mathrm{Si}}-\mathrm{a}_{\mathrm{Ge}}}{\mathrm{a}_{\mathrm{Si}}}\left(\frac{2 c_{12}}{c_{11}}+1\right) x
$$

Since $\Xi_{u}$ is three orders of magnitude larger than $\Delta_{C}$ (with $\Xi_{u} \sim 10 \mathrm{eV}$ and $\Delta_{C} \sim 2 \mathrm{meV}$ ), relatively small changes in $x$ may lead to important shifts in the energies of the valleys. For example, for $\mathrm{Si}$ grown over a relaxed $\mathrm{Si}_{1-x} \mathrm{Ge}_{x}$ alloy with $x=0.2$ on a (001) interface, lattice distortions (smaller than 1\%) lead to a valley strain parameter $\chi \sim-20$. For $\mathrm{Si}_{1-x} \mathrm{Ge}_{x}$ alloys of arbitrary composition, we estimate $\chi(x)=-95 x$. Negative values of $\chi$ indicate tensile strain $\left(a_{\|}>a_{\mathrm{Si}}\right)$, favoring the $z$ valleys energetically. Commensurate growth of $\mathrm{Si}$ over $\mathrm{Si}_{1-x} \mathrm{C}_{x}$ or $\mathrm{SiC}$, which is more difficult experimentally due to the extreme lattice mismatch between the materials, would lead to compressive strain $\left(a_{\|}<a_{\mathrm{Si}}\right)$, so that $\chi>0$. The lattice mismatch between $\mathrm{C}$ (diamond) and $\mathrm{Si}$ is $34 \%(3.57 \AA$ versus $5.43 \AA)$, as compared to $4.2 \%$ for Ge with respect to $\mathrm{Si}\left(5.66 \AA\right.$ versus $5.43 \AA$ ). When we replace $\mathrm{a}_{\mathrm{Ge}}$ by $\mathrm{a}_{\mathrm{C}}$ in Eq. (5)), the estimated valley strain parameter for $\mathrm{Si}$ grown over relaxed $\mathrm{Si}_{1-x} \mathrm{C}_{x}$ would be $\chi \sim 800 x$. This is actually ${ }^{2}$ lower bound for $\chi$, since this alloy's average lattice parameter is somewhat smaller than predicted by Vegard's law. 16

A symmetry argument based on the differentiated fit of the six envelope functions to the Si host geometry and boundary conditions may be used to include the effect of the confinement potentials due to the alloy regions in $\mathrm{Si}_{1-x} \mathrm{Ge}_{x} / \mathrm{Si} / \mathrm{Si}_{1-x} \mathrm{Ge}_{x}$ heterostructures, or due to an interface with any potential barrier (e.g. $\mathrm{Si} / \mathrm{SiO}_{2}$ ) within a few effective Bohr radii from the impurity. Assuming that all Si/barrier interfaces are perpendicular to the growth direction $z$, the components with $F_{ \pm z}$ envelopes are favored energetically, regardless of the value or sign of $\chi$, since the smaller effective Bohr radius $b$ along $z$ guarantees a less significant penetration of the wave function into the barrier regions as compared to the other envelopes with the larger Bohr radius $a$ along $z$. This effect is phenomenologically included in the third term $H_{\mathrm{z}}$ of the donor electron Hamiltonian $H$ through an energy shift $\Delta$. The parameter $\Delta$ is always mositive, and its value depends on the barrier height and on the impurity position with respect to the interface.26 We estimate it here to be up to $\sim 10 \mathrm{meV}$.

In addition to valley energy shifts, the presence of an interface perpendicular to $z$ also leads to coupling between the $\pm z$ valleys. Surface-induced intervalley scattering 27 is introduced phenomenologically in $H_{z}$ through the parameter $\Omega$. This coupling is in general complex, and leads to energy shifts smaller than $1 \mathrm{meV}$ in realistic situations.27.28

\section{Single donor electron states and energy spectrum}

In the absence of strain and other perturbations, the single donor electron Hamiltonian consists only of the valleyorbit coupling, i.e. $H=H_{v o}$. If $\delta=0$, so that all the valley-orbit scattering matrix elements are the same, the eigenstates of $H_{v o}$ are a singlet with energy $-5 \Delta_{C}$ and a quintuplet with energy $\Delta_{C}$. When $\delta \neq 0$, the quintuplet splits into a triplet and a doublet, so that a single donor electron has the state sequence of a singlet, a triplet, and a doublet, which have energies $-(5+\delta) \Delta_{C},(1+\delta) \Delta_{C}$, and $(1-\delta) \Delta_{C}$, respectively. For Si, where $\delta \approx-0.3$ and $\Delta_{C} \approx 2.16 \mathrm{meV}$, the singlet is at $-4.7 \Delta_{C}$, the triplet at $0.7 \Delta_{C}$, and the doublet at $1.3 \Delta_{C}$. The first excited state here 
is a triplet that is $5.4 \Delta_{C} \sim 11.7 \mathrm{meV}$ above the ground singlet state. The valley compositions of these states show that the ground singlet has $A_{1}$ symmetry, corresponding to a symmetric superposition of all six valleys: $\frac{1}{\sqrt{6}}(1,1,1,1,1,1)$, while the triplet and doublet have $T_{1}$ and $E$ symmetries as expected. 19

When the Si lattice is uniaxially strained along the $z$ axis, $H=H_{v o}+H_{\text {strain }}$. Now the degeneracy in the triplet and doublet states are also lifted. For $\chi<0$, the ground state energy is $\Delta_{C}\left[-(2+\delta)+\chi / 2-(3 / 2) \sqrt{\chi^{2}+4 \chi / 3+4}\right]$ : It is an admixture of the $A_{1}$ state with one of the components of $T_{1}$, with valley composition $\left(\alpha_{1}, \alpha_{1}, \alpha_{3}, \alpha_{3}, \alpha_{3}, \alpha_{3}\right)$ and $\alpha_{3}=\alpha_{1} /\left[\sqrt{\chi^{2}+4 \chi / 3+4}-(\chi+2 / 3)\right]$. Notice that the ground state approaches $(1,1,0,0,0,0) / \sqrt{2}$ as $\chi \rightarrow-\infty$. In this limit, the first excited state is one of the original triplet states (in the absence of strain), with energy $\Delta_{C}(1+\delta+2 \chi)$ and valley composition $(1,-1,0,0,0,0) / \sqrt{2}$. The energy splitting between these lowest states approaches $2(1+\delta) \Delta_{C} \sim$ $1.4 \Delta_{C} \sim 3.02 \mathrm{meV}$. For $\chi>0$, the lowest energy states are admixture of $A_{1}$ with the other two components of $T_{1}$ : The (nondegenerate) ground state has energy $\Delta_{C}\left[-(2+\delta)-\chi / 2-(3 / 2) \sqrt{\chi^{2}+4 \chi / 3+4}\right] \sim \Delta_{C}(-3-\delta-\chi)$, while the first excited state (actually a doublet) has energy $\Delta_{C}(1+\delta-\chi)$, with a splitting from the ground state approaching $(4+2 \delta) \Delta_{C} \sim 3.4 \Delta_{C} \sim 7.34 \mathrm{meV}$ as $\chi \rightarrow+\infty$. Hydrostatic (dilation) strain causes a rigid energy shift in the six valleys, keeping the splittings and valley compositions of all eigenstates unchanged. Since our focus here is to understand wavefunction overlaps, we do not consider effects of hydrostatic strain.

In Fig. 2, panel (a), we plot the energy splitting between the first excited state and the ground state of a single donor electron as a function of the valley strain $\chi$. This quantity is relevant in estimating time scales which determine the adiabatic condition in time-dependent processes driven by the gate potential variation. As we have shown above, the energy splitting becomes smaller when the bulk ground and excited states mix to form strained ground and excited states. The variation of these energies can be seen in Fig. 1 of Ref. 23. It is also clear from that figure why the curve we plot here has a cusp at $\chi=0$ : A level crossing occurs at $\chi=0$ for the first excited state.

The strength of the hyperfine coupling between the donor electron and nuclear spins is also important for the $\mathrm{Si}$ QC, since it is invoked in both single qubit and two-qubit operations. 1 In terms of the donor electron state composition, it can be expressed as

$$
A(\chi) / A_{0}=\frac{1}{6}\left|\sum_{\mu} \alpha_{\mu}\right|^{2} \leq 1,
$$

where $A_{0}$ is the value of $A(\chi=0)$. This is a differentiable function of $\chi$, so its peak at $\chi=0$ is smooth, as is shown in panel (b) of Fig. 2. Furthermore, for $\chi \rightarrow-\infty$, the ground state composition approaches $1 / \sqrt{2}(1,1,0,0,0,0)$, so that $A(-\infty) / A_{0}=1 / 3$; while for $\chi \rightarrow \infty$, the ground state approaches $1 / 2(0,0,1,1,1,1)$, and $A(\infty) / A_{0}=2 / 3$. The curve in Fig. 2(b) clearly approaches these two limits. Results summarized in Fig. 2 indicate that donors in uniaxially strained Si will have "inferior" single-electron properties compared to the relaxed material for the purpose of QC operations, requiring longer times to perform an adiabatic evolution of the electron state and providing a reduced hyperfine coupling.

When the $z$-direction confinement is added, it introduces very small energy shifts and additional off-diagonal coupling between $z$ and $-z$ directions. In the presence of $H_{v o}$ and/or $H_{\text {strain }}$, the effects of $H_{z}$ are negligible for all relevant properties discussed here. Thus we do not include this term in our calculation below.

We emphasize the following general properties of the spectrum of $H$ in Eq. (3) when $\Delta_{C}>0$ : (i) the ground state is always non-degenerate, and (ii) the ground state valley populations in opposite symmetry directions, e.g. $\mu=1, \nu=2$, always satisfy $\left|\alpha_{\mu}\right|^{2}=\left|\alpha_{\nu}\right|^{2}$.

\section{Heitler-London approximation}

In this paper we use Heitler-London (HL) approximation to calculate the inter-donor exchange coupling as the energy difference between the two-electron singlet and triplet states 29 Considering a pair of donors at $\mathbf{R}_{A}=0$ and $\mathbf{R}_{B}=\mathbf{R}$ and assuming $R=|\mathbf{R}| \gg a, b$ (the effective Bohr radii), the energies of the singlet and triplet states are

$$
E_{s}=2 E_{0}+\frac{H_{0} \pm H_{1}}{1 \pm S^{2}}
$$

where $E_{0}$ is the single impurity electronic energy, while $H_{0}, H_{1}$, and $S$ are integrals involving two electronic wave functions of the form (2) centered at the origin and $\mathbf{R}$, and are given in the Appendix. Contrary to the case of the $\mathrm{H}_{2}$ molecule, where these integrals are isotropic and smoothly decaying functions of the relative distance $R$, in the case 
of Si they are extremely anisotropic and sensitive functions of $\mathbf{R} 11$ The HL expression for the exchange splitting in $\mathrm{Si}$ is

$$
\begin{aligned}
J(\mathbf{R}) & =E_{t}-E_{s} \\
& =\sum_{\mu, \nu}\left[\sum_{\mathbf{K}, \mathbf{K}^{\prime}}\left|c_{\mathbf{K}}^{\mu}\right|^{2}\left|c_{\mathbf{K}^{\prime}}^{\nu}\right|^{2} e^{i\left(\mathbf{K}-\mathbf{K}^{\prime}\right) \cdot \mathbf{R}}\right]\left|\alpha_{\mu}\right|^{2}\left|\alpha_{\nu}\right|^{2} \mathcal{J}_{\mu \nu}(\mathbf{R}) \cos \left(\mathbf{k}_{\mu}-\mathbf{k}_{\nu}\right) \cdot \mathbf{R},
\end{aligned}
$$

which is derived in the Appendix, where the explicit expression for $\mathcal{J}_{\mu \nu}(\mathbf{R})$ is also given. The second summation (the part within the square bracket) in (8) refers to the reciprocal lattice expansion of the periodic part of the Bloch function, $u_{\mu}(\mathbf{r})=\sum_{\mathbf{K}} c_{\mathbf{K}}^{\mu} e^{i \mathbf{K} \cdot \mathbf{r}}$, and is identically unity when $\mathbf{R}$ is an fcc crystal lattice vector.

A prominent feature of the above expression for the exchange coupling $J(\mathbf{R})$ is the presence of the fast oscillatory terms $\cos \left(\mathbf{k}_{\mu}-\mathbf{k}_{\nu}\right) \cdot \mathbf{R}$, which are periodic with wavelengths of the order of the atomic spacing $(\sim 5 \AA$ in $\mathrm{Si})$. The coefficients $\mathcal{J}_{\mu \nu}(\mathbf{R})$ are slowly varying functions of $\mathbf{R}$ as they are integrals containing the envelope functions $F_{\mu}(\mathbf{R})$, whose characteristic decay length is the effective Bohr radius of the order of $20 \AA$.

\section{RESULTS}

We have performed HL calculations of the donor electron exchange for different configurations of a pair of donors. Results (Figs. 346) presented here are obtained by taking the summation over reciprocal lattice vectors to be unity: $\sum_{\mathbf{K}, \mathbf{K}^{\prime}}\left|c_{\mathbf{K}}^{\mu}\right|^{2}\left|c_{\mathbf{K}^{\prime}}^{\nu}\right|^{2} e^{i\left(\mathbf{K}-\mathbf{K}^{\prime}\right) \cdot \mathbf{R}}=1$, i.e. the term within the square bracket in Eq. (8) is taken to be unity which is equivalent to calculating $J(\mathbf{R})$ only for those values of $\mathbf{R}$ which correspond precisely to $\mathbf{R}$ being an fcc lattice vector. The continuous lines shown in the figures correspond to the free-electron limit $c_{\mathbf{K}}^{\mu} \approx \delta_{\mathbf{K}, 0}$, which is a first order approximation to the lowest conduction band in homopolar semiconductors.30 We investigate the exchange coupling in uniaxially strained $\mathrm{Si}$ as we vary the inter-donor distance, the inter-donor direction relative to the strain axis (defined to be the $z$ axis), and the sign and the magnitude of strain itself. Results presented in Figs. 3 to 6 give a comprehensive account of the general trends and effects to be expected, providing new qualitative and quantitative insight into the problem of donor electron exchange coupling in multivalley semiconductors.

In Fig. 3 we plot the exchange coupling $J$ as a function of the inter-donor distance $\mathbf{R}$ and valley strain $\chi$ for a pair of donors along the [100] axis. Notice that as $\chi$ increases from negative to positive values, the exchange coupling becomes increasingly oscillatory. Such behavior can be easily understood from the expression of $J(\mathbf{R})$ in Eq. (8) and from the donor ground state valley composition. For $\chi \ll 0$ with strain along the $z$ axis, the dominant components of the ground state are

$$
\psi(\mathbf{r}) \sim \alpha_{1} F_{z} e^{i \mathbf{k}_{z} \cdot \mathbf{r}}+\alpha_{2} F_{-z} e^{-i \mathbf{k}_{z} \cdot \mathbf{r}},
$$

where $\alpha_{1}=\alpha_{2} \sim 1 / \sqrt{2}$, so that the largest oscillatory contribution to $J(\mathbf{R})$ comes from terms with factors $\cos \left(k_{z} \pm\right.$ $\left.k_{-z}\right) R_{z}$ with $k_{-z}=-k_{z}$. The only effect of varying the $x$-component of $\mathbf{R}$ is to vary the coefficients $\mathcal{J}_{\mu \nu}$, which are slowly decaying functions of $|\mathbf{R}|$. As $\chi$ increases toward zero, the contribution from the four components $\alpha_{3}, \ldots, \alpha_{6}$ grows, and fast oscillations are superimposed on the overall decaying envelope of $J\left(R_{x}\right)$. For $\chi \gg 0$, these four components dominate the ground state valley composition, and the oscillations are enhanced with respect to relaxed bulk Si.

From the perspective of predicting and controlling the donor pair exchange coupling, results in Fig. 3 indicate that the optimal degree of strain is in the range $\chi \lesssim-5$, since this leads to a simple hydrogenic-type behavior for the particular type of relative positions (along [100] axis) of a pair of donors, as was presumed in the original $\mathrm{Si}$ and $\mathrm{Si} / \mathrm{Ge}$ QC proposals. relaxed bulk $\mathrm{Si}$, a feature with considerable practical advantages in terms of increasing the speed of gate operations in the QC.

To study cases when the two donors are not positioned along the [100] axis, we first choose a reference configuration, with a donor pair along [100] direction. We choose to work with uniaxially strained Si with $\chi=-20$ from now on as it corresponds to the realistic situation of $\mathrm{Si}$ grown over relaxed $\mathrm{Si}_{0.8} \mathrm{Ge}_{0.2}$. We arbitrarily pick the relative position $R_{x} \sim 110 \AA$ marked by the solid triangle in Fig. 3, with respect to which various displacements are considered for one of the two donors. In Fig. $⿴$ we plot the exchange coupling $J$ as one of the donors is displaced from the reference site along either the $y([010])$ or the $z([001])$ axis. The upper curve, corresponding to displacements along the $y$ axis, reflects essentially the same qualitative behavior as in Fig. 3 for $\chi=-20$. However, when the relative displacement $\mathbf{R}$ acquires any nonzero $R_{z}$ component, exchange becomes rapidly oscillatory, as indicated by the triangles in the lower curve. The same trends are seen in Fig. 5, where displacements along the [110], [011] and [101] directions are 
considered. Among those, only displacements along the [110] diagonal direction lead to a monotonous variation of $J$ with distance without any oscillations. The other two types of displacement involve a nonzero $R_{z}$ component in the relative position $\mathbf{R}$, leading to fast oscillations in the exchange energy.

The overall effect of uniaxial strain in the exchange coupling of a donor pair in the $x-y$ plane is summarized in Fig. 6. There we consider all possible in-plane substitutional donor pairs with inter-donor distances in the range of $90 \AA<R<180 \AA$. For impurity pairs in uniaxially strained Si $(\chi=-20)$, the exchange is a single-valued function of $R$, decaying smoothly with distance, while for unstrained $\mathrm{Si}(\chi=0)$ the exchange is significantly reduced with respect to the strained results, and is sensitively dependent on the relative position $\mathbf{R}$.

Notice that in all the results presented here the oscillatory behavior of $J$ is almost always accompanied by partial cancellations, meaning that in the oscillatory regime, the values of $J$ are always smaller than in the corresponding non-oscillatory situations. This is clearly evident in Fig. 3, comparing curves with increasing values of $\chi$; or in Figs. A and 5, comparing curves with zero and nonzero $R_{z}$ components for the same inter-donor distance $|\mathbf{R}|$. This result can be mathematically confirmed from expression (\$), where the $\cos \left(\mathbf{k}_{\mu}-\mathbf{k}_{\nu}\right) \cdot \mathbf{R}$ factors are responsible both for oscillations and for partial cancellations $\left[\mathcal{J}_{\mu \nu}(\mathbf{R})>0\right.$ for arbitrary $\mu$ and $\left.\nu\right]$. It is somewhat different (and perhaps counterintuitive) from an ordinary two-wave interference. One aspect of our results presented in the figures (Figs. 3 . 6) that needs to be emphasized is that our exact HL calculations are done only for discrete values of the relative displacement or inter-donor distance, and the smooth curves are guides to the eye. As mentioned in the beginning of this section, the smooth continuous curves in our results correspond to an effective free electron approximation.

\section{SUMMARY AND DISCUSSIONS}

\section{A. Approximations}

We now briefly review the main approximations involved in the theoretical approach adopted in this paper, and discuss various possible improvements. In our perturbation theory calculation, the only effect of strain is to change the valley populations of the donor electron states through the diagonal elements of the six-valley Hamiltonian $H$. We do not consider strain-dependent changes in the effective masses, the inter-valley scattering matrix elements $\Delta_{c}$ and $\delta$, or the BZ locations for the conduction band minima. Since the lattice parameter changes are quite small-less than $1 \%$ as we mentioned in the beginning of Sect. II, we believe that our approach is well-suited for the modestly strained systems considered here. For highly strained systems (such as $\mathrm{Si}$ grown on $\mathrm{Si}_{1-x} \mathrm{C}_{x}$ with high concentration of carbon), the separation of $H$ into $H_{v o}$ and $H_{\text {strain }}$ may not be justifiable, and all matrix elements should be consistently assessed to determine how they depend on strain.

In our envelope function approach, we have adopted the variational form and parameters obtained from the onevalley Kohn-Luttinger treatment. Calculations incorporating the valley-orbit interactions and strain in the original Hamiltonian, and treating the effective Bohr radii $a$ and $b$ and the valley compositions as independent variational parameters (imposing symmetry and normalization constrains), would have led to a better quantitative description of the ground state binding energy and possibly the wave function. Such calculations for donorsip Si have been performed to determine the binding energies using only spherically symmetric trial wave functions, 21,31 which is not adequate for the present study. Our interest in this paper is not in obtaining extremely accurate donor binding energies, but in calculating reasonably good wavefunctions so as to obtain reliable donor exchange energies.

Atomistic ab initio studies based on density-functional schemes can overcome the limitatipns of the envelopefunction/effective-mass theory and have been employed in the literature to study effects of strain 13 and alloy disorder. 16 The difficulty in describing a single shallow impurity originates from the underlying supercell approach with periodic boundary conditions, which leads to spurious effects due to interactions between the impurity and its periodic replicas, Extrapolation schemes towards an infinite supercell have been proposed for the single impurity binding energy, 32 although periodic boundary conditions cause additional difficulties for a reliable description of the asymptotic behavior of the wave functions.

The HL approximation adopted in this paper is the simplest approach to calculate exchange coupling in a variety

of situations, such as atomic lattices 33 random donor arrays, 34 and double quantum dots.35. 34 Improvement can be made through various ways. For instance, it is quite common to use the valence bond theory (also called the molecular orbital theory) or the configuration interaction (often combined with a self-consistent mean field calculation) approach to ohtain mpre accurate spectra (and thus exchange interaction when specifically pursued) for molecules or quantum dots.29 3538 For donor arrays in $\mathrm{Si}$ (relaxed or strained), such calculations will be complicated by the Si band structure. However, as we have shown in the current study, as long as the effective Bohr radius is much larger than the lattice spacing, one can separate the fast oscillating (due to the Bloch functions) parts of integrals for the matrix elements from the slowly varying envelope function parts, thus simplifying the calculation. Indeed, there should be 
no significant theoretical difficulty for a more complete molecular orbital calculation for donors in Si. In the current study, our focus is on the qualitative behavior of the exchange coupling between two donors, therefore we have not attempted to improve upon the HL approximation. This can be easily done following earlier calculations in GaAs quantum dots 38 if the experimental development warrants such improvements of the theory. In addition, the proposed Si QC would operate under a uniform magnetic field of 1 to 2 Tesla applied along the $z$ axis (see Fig. 1). Previous studies 38 indicate that uniform magnetic fields of up to 2 Tesla have no qualitative effect in the exchange coupling of electrons in double quantum dot bound states. For a pair of donors, a reduction in $J$ due to the shrinking of the envelopes perpendicular to the magnetic field direction and magnetic phase interference should be expected, but this should not lead to any qualitatively different effect.

As we have mentioned before, in our calculation we have approximated the Bloch functions by the corresponding plane wave functions, which is in essence a free electron approximation for the conduction band of Si.30 The approximation here amounts to keeping only the $c_{0}$ term (and let $c_{0}=1$ ) in the reciprocal lattice expansion of the periodic part of the Bloch functions. The plane wave approximation actually obtains the exact results for lattice points on the same fcc lattice, where $e^{i\left(\mathbf{K}-\mathbf{K}^{\prime}\right) \cdot \mathbf{R}}=1$ so that the sums over reciprocal lattice vectors $\mathbf{K}$ are normalized to 1 . However, for an arbitrary $\mathbf{R}$, our calculation should mostly give an upper bound to an exact HL calculation. If the $c_{\mathbf{K}}$ coefficients have a broad distribution, the sums would tend to be much smaller since they involve the fourth power of $c_{\mathbf{K}}$ as is shown in Eq. (8). Thus the continuous lines in our figures (Fig. 3 to Fig. (7) should be taken more as guides to the eye than as the actual values of exchange, which can be much smaller. As mentioned before, we carry out our exact Heitler-London exchange coupling calculations only for discrete values of inter-donor separation (triangles, squares, etc. in the figures) whereas the continuous curves represent the free electron HL approximation. Further calculations are underway to go beyond the free electron approximation to address this issue, and those results will be presented elsewhere.

\section{B. Valley degeneracy effects}

Our results for Si uniaxially strained along the $z$ axis indicate that, for $\chi \lesssim-5$, inaccuracies in the positioning of substitutional ${ }^{31} \mathrm{P}$ donor pairs within the $x-y$ plane produce only small effects in the electron exchange coupling. Naturally, the next question is whether it is possible to avoid the oscillatory behavior due to lattice-parameter-scale inaccuracies in the $R_{z}$ component of the relative position between a pair of donors. As noted in Sec. III, these oscillations in $J(\mathbf{R})$ are due to the specific form of the ground state wave function (9), which dictates that the contribution from the two valley minima are equally important. This result is in turn a consequence of the degeneracy of the $\mathbf{k}_{z}$ and $-\mathbf{k}_{z}$ valleys in bulk $\mathrm{Si}$, whose energies are lowered equally with respect to the four perpendicular valleys by uniaxial tensile strain. The same effect occurs in the two-dimensional electron gas (2DEG) in (001) $\mathrm{Si} / \mathrm{SiO}_{2}$ inversion layers 39 and $\mathrm{Si} / \mathrm{Si}_{1-x} \mathrm{Ge}_{x}$ quantum wells 28 where, due to the confining potential at the interface, the lowest electronic subbands originate from the bulk $\mathrm{Si}_{z}$ and $-\mathbf{k}_{z}$ valleys alone. It has been shown theoretically 27,10 and experimentally 283 that this valley degeneracy may be lifted in heterostructure-type configurations, leading to experimental valley splittings of the order of $0.1 \mathrm{meV}$. Such splittings refer to the unperturbed doubly-degenerate ground states, and the repulting singlet ground state is not one of the $\mathbf{k}_{z}$ and $-\mathbf{k}_{z}$ valleys, but an equally weighted admixture of these states 27 In the present case, $H_{v o}$ alone leads to a nondegenerate ground state, which is $12 \mathrm{meV}$ below the first excited state. Under the $H_{\text {strain }}$ perturbation, the ground state remains nondegenerate, with an energy separation to the first excited state reduced, but still larger than $3 \mathrm{meV}$. Further perturbations, e.g. $H_{z}$ that produces the valley splitting observed in the 2DEG, have negligible quantitative impact in any of the results obtained here. Moreover, perturbations of the form $H_{z}$ always lead to an equally weighted admixture of the $\mathbf{k}_{z}$ and $-\mathbf{k}_{z}$ valleys, thus donor electron valley composition always has contributions from these two valleys with the same weight, i.e. $\left|\alpha_{1}\right|=\left|\alpha_{2}\right|$ in (9).

We note that for Ge, where four conduction band minima occur at the inequivalent $L$ points in the BZ, $\langle 111\rangle$ tensile strain leads to a nondegenerate band minimum. Since the lattice parameter of Ge is larger than that of $\mathrm{Si}$ and $\mathrm{C}$ this possibility within group-IV semiconductors remains restricted to Ge-rich strained alloys grown over relaxed Ge,t with the additional complication of bringing alloy disorder effects into the non-degenerate ground state layer where the donors are placed. We do not consider Ge in this paper since there is no particular experimental effort directed toward the design of a Ge-based QC. 


\section{Estimates for static electric field effects}

Static electric fields generated by the top gates can be used to slightly modify the donor electron wavefunction in its shape and weight distribution, so that such fields can be used to overcome lattice-parameter-scale inaccuracies in the $R_{z}$ component of the relative position of a pair of substitutional impurities. A crude estimate of the- uniform static electric field effects in the donor bound states may be inferred from results for the hydrogen atom. 12 To the first order in the field, the $\mathrm{H}$ ground state wave function in the presence of a uniform electric field $\mathcal{E}$ along $z=r \cos \theta$ is given by

$$
\psi_{\mathrm{H}}(\mathbf{r})=\frac{1}{\sqrt{\pi a_{0}^{3}}} e^{-r / a_{0}}\left[1-\frac{\mathcal{E}}{e}\left(a_{0} r+\frac{r^{2}}{2}\right) \cos \theta\right],
$$

where $a_{0}=0.529 \AA$ is the Bohr radius, and $e$ is the electron charge. The expectation value of the electron position is thus shifted by the field from $\langle\mathbf{r}\rangle=0$ to $\langle\mathbf{r}\rangle_{\mathrm{H}}=-\frac{9}{2} a_{0}^{3} \overrightarrow{\mathcal{E}} / e$, which corresponds to an average displacement of $4.63 \times 10^{-6} \AA$ for $\mathcal{E}=10 \mathrm{kV} / \mathrm{cm}$. This negligible shift is greatly enhanced for donor bound states in Si. Assuming a spherically averaged envelope with an effective Bohr radius $a^{*}=\left(a^{2} b\right)^{1 / 3}$, we get $\langle\mathbf{r}\rangle_{\mathrm{Si}}=\epsilon\left(a^{*} / a_{0}\right)^{3}\langle\mathbf{r}\rangle_{\mathrm{H}} \cong 7.4 \times 10^{5}\langle\mathbf{r}\rangle_{\mathrm{H}}$, where $\epsilon=12.1$ it the $\mathrm{Si}$ dielectric constant. An average position shift of one monolayer $\left(1 \mathrm{ML}=\mathrm{a}_{\mathrm{Si}} / 2=2.7 \AA\right.$ in $\left.\mathrm{Si}\right)$ thus corresponds to $\mathcal{E}=8 \mathrm{kV} / \mathrm{cm}$. In the current context, it is plausible to expect that a uniform electric field of tens of $\mathrm{kV} / \mathrm{cm}$ may compensate for atomic misplacements of a few monolayers along the field direction.

It is interesting to note that, according to Eq.(10), the applied field would have no first-order effect on the electronnucleus hyperfine coupling, which is proportional to the electronic density at the nuclear site, $A \sim|\psi(0)|^{2}$. Since the A-gates are primarily meant to reduce this coupling by pulling the electron wavefunction away from the donor towards the barrier, fields much stronger than those quoted above are required to perform such operations. In the strong field limit, donor ionization starts to take place. The field dependence of the dissociation rate for atomic $\mathrm{H}$ is 43

$$
\frac{\Delta N}{T} \propto \mathcal{E}^{1 / 4} \exp \left(-\frac{2 e}{3 \mathcal{E} a_{0}^{2}}\right)
$$

which means that dissociation becomes appreciable for field values in the order of $\mathcal{E}_{\mathrm{H}}=e / a_{0}^{2}=5 \times 10^{6} \mathrm{kV} / \mathrm{cm}$. Under the spherically averaged envelope assumptions above, we estimate that donor ionization in Si would require fields of the order of $\mathcal{E}_{\mathrm{Si}}=\left[\left(a_{0} / a^{*}\right)^{2} / \epsilon\right] \mathcal{E}_{\mathrm{H}} \cong 5.3 \times 10^{-5} \mathcal{E}_{\mathrm{H}} \sim 300 \mathrm{kV} / \mathrm{cm}$, which is one order of magnitude larger than the fields required to compensate for atomic displacements of a few ML.

\section{Fabrication-related aspects}

We have shown in this paper that strained Si grown commensurately over relaxed $\mathrm{Si}_{1-x} \mathrm{Ge}_{x}(001)$ alloys presents clear theoretical advantages over relaxed $\mathrm{Si}$ as a host material for ${ }^{31} \mathrm{P}$ donor impurities in Si-based QC architectures. Since the same is not true for strained $\mathrm{Si}$ grown on $\mathrm{Si}_{1-x} \mathrm{C}_{x}$ substrates, here we focus on growth of strained $\mathrm{Si}$ over relaxed $\mathrm{Si}_{1-x} \mathrm{Ge}_{x}$ alloys. One important limiting factor in the fabrication of strained Si layers is the so-called critical thickness, $h_{C}$, the thickness above which the strained layer relaxes by forming topological defects, typically misfit dislocations which originate at the interface and propagate into the bulk. 12 Theoretical estimates through an energy minimization criterion 15 show a sharp decay of $h_{C}$ with the alloy Ge content, from over $1000 \AA$ for $x=0.05$ to 100 $\AA$ for $x=0.35$. Recent advances in epitaxial growth of Si/Ge heterostrucfures have led to the fabrication of samples of $100 \AA$ thick Si layers over relaxed $\mathrm{Si}_{1-x} \mathrm{Ge}_{x}$ alloys with $x$ up to 0.314 with interfaces of extremely good quality and free of dislocations. Also, $150 \AA$ thick layers over $x=0.2$ alloys were recently produced. 15 In both cases, the layer thickness is below (but very close to) the theoretical upper bound for $h_{C}$.

Substrate layers with relatively high Ge-content $(x \sim 0.2)$ serve two useful purposes in the context of QC architecture: (i) the strained Si layer leads to improved behavior of exchange coupling between donor impurity states; (ji) the relaxed alloy layer serves as a barrier separating the Si host containing ${ }^{31} \mathrm{P}$ donors from the conducting gates. values of alloy composition $x$, leading to valley strain $\chi \sim-20$, also impose some limitations in the implementation of the Si-based QC. The magnitude of strain means an upper bound of around $200 \AA$ for the thickness of the strained Si layer, a reduction in the hyperfine coupling, and a reduction in the energy splitting between the ground and the first excited state by a factor of 3 with respect to the relaxed $\mathrm{Si}$ architectures. Alloys with lower Ge content $(x \sim 0.05)$ with valley strain parameter $\chi \sim-5$ provide comparable electronic performance (actually somewhat improved performance in terms of the strength of the hyperfine coupling) and greatly enhanced values of $h_{C}$, over $1000 \AA$. This would obviously facilitate most aspects of the design and fabrication of the Si layers. On the other hand, the low-Ge-content 
alloys do not provide a sufficiently high potential barrier, thus one would have to use the more conventional $\mathrm{SiO}_{2}$ barriers between the strained $\mathrm{Si}$ and the top gates.

Our results refer to structures of strained Si commensurately grown over relaxed random alloys. The inevitable substrate alloy disorder can pose some potential difficulties. Indeed, some randomness in the lattice spacing and thus in strain is bound to occur at the alloy/Si interface. However, this effect quickly decays inside the Si layer. Since we consider donors located as far as $100 \AA$ ( twenty lattice spacings in Si) away from the interface, any alloy disorder effect should be negligible, leading to a uniformly strained Si host for all practical purposes. On the other hand, if a device is based on an heterostructure predominantly formed by $\mathrm{Si}_{1-x} \mathrm{Ge}_{x} / \mathrm{Si}_{1-y} \mathrm{Ge}_{y}$ strained layers (alloy on alloy), as considered in Ref. A, and the donors are located inside an alloy layer (see Fig. Ii (b)), random strain and local chemical disorder would presumably play a much more important role in determining the donor electronic properties, including exchange and hyperfine couplings. The electronic properties of a single impurity is sensitive to local composition fluctuations, which will sertainly influence the performance and reproducibility in the fabrication of such devices. Another suggested alloy $5 \mathrm{Si}_{1-x} \mathrm{C}_{x}$, presents a much larger lattice mismatch within the alloy layer, leading to a much stronger randomness in local strain, which implies a higher probability of defects and smaller critical thickness if one attempts to grow strained Si on top of such a substrate.

We have demonstrated that it is possible to achieve hydrogenic behavior for substitutional donors positioned exactly in the same (001) lattice plane parallel to the interface with the alloy substrate. For the recently proposed 10 "bottomup" approach for Si QC fabrication, in which MBE growth is followed by positioning individual P impurity on the surface with the help of an STM tip, our result implies that small displacements of the P atoms due to surface diffusion or hops among the $\mathrm{Si}$ atoms forming dimers at the $\mathrm{Si}(001) 2 \times 1$ surface should be acceptable for the reliability of QC operations mediated by exchange coupling. The final stage of the "bottom-up" approach involves Si overgrowth, encapsulating the deposited $\mathrm{P}$ donors. This is a crucial step for either relaxed or strained $\mathrm{Si}$, since any atomic-scale $z$-component added to the relative position $\mathbf{R}$ leads to oscillatory behavior and possibly strong reduction of $J$. In MBE growth, surface binding and elastic energy differences among the various species lead to segregation effects, driving $\mathrm{P}$ atoms from previously grown layers into newly deposited ones. Segregation may be reduced by lowering the growth temperature and by increasing the growth rate, 111 but it may not be eliminated entirely. In a different, "top-down", approach, the P array in Si is formed by ion-implantation techniques, which implies a low degree of control over $R_{z}$ on a lattice-parameter length scale. Residual variations in the relative positions of the donors must be corrected by individually calibrating the A and J gates. In other words, one must rely on probing and "correcting" each donor bound state through the surface gates. In general, our work establishes that Si QC design could tolerate some imprecision in the positioning of the dopant atoms in a 2D layer parallel to the interface, but any lack of precise control in the $z$ direction could be fatal.

We point out an additional advantage of strained over relaxed Si according to our numerical results. Following all overgrowth stages, one A gate must be placed on top of each buried impurity and one $\mathrm{J}$ gate in-between neighboring impurity pairs (see Fig. 1(a)). We have shown that the exchange coupling is enhanced for donor pairs parallel to the interface in negatively strained Si configurations. This allows for larger inter-donor distances as compared to relaxed $\mathrm{Si}$, which is particularly convenient in terms of fabricating and accurately positioning the external gates.

Finally, we point out the obvious fact that, although we have emphasized the relevance of our theory to Si QC architectures throughout this paper, our work is of general validity completely transcending $\mathrm{QC}$ considerations and context. In particular, we have developed a theory for calculating the donor exchange energy in strained Si systems incorporating the quantum interference effect arising from the multiple valley structure of Si conduction band. Our results should apply to any problem involving Si donor exchange coupling considerations.

\section{ACKNOWLEDGMENTS}

We thank Rogerio de Sousa for calling our attention to the approximate Heitler-London form for the exchange coupling we used previously. We also thank Bruce Kane, Ravin Bhatt, and Michelle Simmons for useful conversations. This work is supported by ARDA and NSA. BK acknowledges financial support from CNPq (Brazil).

\section{APPENDIX A: HEITLER-LONDON APPROXIMATION FOR DONORS IN SI}

In this paper the Heitler-London approximation is employed to calculate the exchange splitting $J$ between the ground singlet and triplet states for two ${ }^{31} \mathrm{P}$ donors embedded in strained Si. Here we derive the expression for $J$ in terms of the donor relative position and the host band structure parameters. Assuming the ground donor electron wave functions to be $\psi(\mathbf{r})$, the singlet and triplet wave functions for donors located at the origin and $\mathbf{R}$ are 


$$
\begin{aligned}
& \psi_{s}\left(\mathbf{r}_{1}, \mathbf{r}_{2}\right)=\frac{1}{\sqrt{2\left(1+S^{2}\right)}}\left[\psi\left(\mathbf{r}_{1}\right) \psi\left(\mathbf{r}_{2}-\mathbf{R}\right)+\psi\left(\mathbf{r}_{1}-\mathbf{R}\right) \psi\left(\mathbf{r}_{2}\right)\right] \times \frac{1}{\sqrt{2}}(|\uparrow \downarrow\rangle-|\downarrow \uparrow\rangle), \\
& \psi_{t}\left(\mathbf{r}_{1}, \mathbf{r}_{2}\right)=\frac{1}{\sqrt{2\left(1-S^{2}\right)}}\left[\psi\left(\mathbf{r}_{1}\right) \psi\left(\mathbf{r}_{2}-\mathbf{R}\right)-\psi\left(\mathbf{r}_{1}-\mathbf{R}\right) \psi\left(\mathbf{r}_{2}\right)\right] \times \frac{1}{\sqrt{2}}(|\uparrow \downarrow\rangle+|\downarrow \uparrow\rangle),
\end{aligned}
$$

where $S$ is the overlap integral

$$
S=\int d^{3} \mathbf{r} \psi^{*}(\mathbf{r}) \psi(\mathbf{r}-\mathbf{R}) .
$$

The Hamiltonian that governs this two-electron system is

$$
H=\sum_{i=1}^{2}\left\{K_{i}-\frac{e^{2}}{\epsilon}\left[\frac{1}{\left|\mathbf{r}_{i}\right|}+\frac{1}{\left|\mathbf{r}_{i}-\mathbf{R}\right|}\right]\right\}+\frac{e^{2}}{\epsilon}\left(\frac{1}{\left|\mathbf{r}_{1}-\mathbf{r}_{2}\right|}+\frac{1}{R}\right) .
$$

The energy splitting between the ground singlet and triplet states within the Heitler-London approximation is 29

$$
\begin{aligned}
J(\mathbf{R}) & =E_{t}-E_{s}=\left\langle\psi_{T}|H| \psi_{T}\right\rangle-\left\langle\psi_{S}|H| \psi_{S}\right\rangle \\
& =\frac{2}{1-S^{4}}\left(H_{0} S^{2}-H_{1}\right) .
\end{aligned}
$$

The terms appearing both here and in (7) are

$$
\begin{aligned}
& H_{0}=\int d^{3} \mathbf{r}_{1} d^{3} \mathbf{r}_{2}\left|\psi^{*}\left(\mathbf{r}_{1}\right)\right|^{2} \mathcal{O}\left|\psi^{*}\left(\mathbf{r}_{2}-\mathbf{R}\right)\right|^{2}, \\
& H_{1}=\int d^{3} \mathbf{r}_{1} d^{3} \mathbf{r}_{2} \psi^{*}\left(\mathbf{r}_{1}\right) \psi^{*}\left(\mathbf{r}_{2}-\mathbf{R}\right) \mathcal{O} \psi\left(\mathbf{r}_{1}-\mathbf{R}\right) \psi\left(\mathbf{r}_{2}\right),
\end{aligned}
$$

with

$$
\mathcal{O}=\frac{e^{2}}{\epsilon}\left(-\frac{1}{\left|\mathbf{r}_{1}-\mathbf{R}\right|}-\frac{1}{\left|\mathbf{r}_{2}\right|}+\frac{1}{\left|\mathbf{r}_{1}-\mathbf{r}_{2}\right|}+\frac{1}{R}\right)
$$

Replacing the ground state wave functions of the form (2) for $\psi$ in the expressions above leads to sums over integrals which involve different envelope functions and phases coming from the Bloch wave functions. For example, for the overlap (A1) one gets

$$
\begin{aligned}
S & =\sum_{\mu \nu \mathbf{K} \mathbf{K}^{\prime}} A_{\mu \nu \mathbf{K} \mathbf{K}^{\prime}} \int d^{3} \mathbf{r}_{1} F_{\mu}\left(\mathbf{r}_{1}\right) F_{\nu}\left(\mathbf{r}_{1}-\mathbf{R}\right) e^{-i\left(\mathbf{k}_{\mu}+\mathbf{K}\right) \cdot \mathbf{r}_{1}} e^{i\left(\mathbf{k}_{\nu}+\mathbf{K}^{\prime}\right) \cdot\left(\mathbf{r}_{1}-\mathbf{R}\right)} \\
& =\sum_{\mu \nu \mathbf{K} \mathbf{K}^{\prime}} A_{\mu \nu \mathbf{K K} \mathbf{K}^{\prime}} e^{-i \mathbf{k}_{\nu} \cdot \mathbf{R}} \int d^{3} \mathbf{r}_{1} F_{\mu}\left(\mathbf{r}_{1}\right) F_{\nu}\left(\mathbf{r}_{1}-\mathbf{R}\right) e^{i\left(\mathbf{k}_{\nu}-\mathbf{k}_{\mu}+\mathbf{K}^{\prime}-\mathbf{K}\right) \cdot \mathbf{r}_{1}}
\end{aligned}
$$

where

$$
A_{\mu \nu \mathbf{K K}}=\alpha_{\mu}^{*} \alpha_{\nu} c_{\mathbf{K}}^{\mu *} c_{\mathbf{K}^{\prime}}^{\nu} .
$$

Following the approximations suggested in Ref. 34, we neglect all integrals which contain rapidly oscillating phases in the integrated variables $\mathbf{r}_{1}$ or $\mathbf{r}_{2}$. The remaining integrals involve different anisotropic envelope functions, and may be cast into forms similar to those appearing in the $\mathrm{H}_{2}$ molecule $\mathrm{HL}$ problem through a coordinate rescaling along the three Cartesian directions by the respective effective Bohr radii. For the specific example above, we get:

$$
S=\sum_{\mu \mathbf{K}} A_{\mu \mu \mathbf{K K}} s_{\mu} e^{-i \mathbf{k}_{\mu} \cdot \mathbf{R}}
$$

with

$$
s_{\mu}=e^{-\mathcal{R}_{\mu}}\left(1+\mathcal{R}_{\mu}+\mathcal{R}_{\mu}^{2} / 3\right) .
$$


Equation $(\mathrm{A} 8 \mathrm{l})$ is exactly the hydrogenic expression for the overlap, but evaluated at the rescaled internuclear distance which, for $\mu=z$, is $\boldsymbol{\mathcal { R }}_{z}=\left(R_{x} / a, R_{y} / a, R_{z} / b\right)$. Integrals appearing in (A3) and (A4) contain a distance-dependent denominator which, at large internuclear separations, may be replaced by the value appropriate for the line joining the two donors. 34 For these terms it is convenient to define

$$
f\left(\theta_{\mu}\right)=\frac{e^{2}}{\epsilon a}\left[\frac{a^{2}}{b^{2}} \cos ^{2} \theta_{\mu}+\sin ^{2} \theta_{\mu}\right]^{1 / 2}
$$

where $\theta_{\mu}$ is the polar angle between the $\mu$-direction and $\mathbf{R}$.

Taking all these considerations into account, we arrive at the final expression for the exchange coupling $J(\mathbf{R})$ :

$$
J(\mathbf{R})=\sum_{\mu, \nu}\left[\sum_{\mathbf{K}, \mathbf{K}^{\prime}}\left|c_{\mathbf{K}}^{\mu}\right|^{2}\left|c_{\mathbf{K}^{\prime}}^{\nu}\right|^{2} e^{i\left(\mathbf{K}-\mathbf{K}^{\prime}\right) \cdot \mathbf{R}}\right]\left|\alpha_{\mu}\right|^{2}\left|\alpha_{\nu}\right|^{2} \mathcal{J}_{\mu \nu}(\mathbf{R}) \cos \left(\mathbf{k}_{\mu}-\mathbf{k}_{\nu}\right) \cdot \mathbf{R} .
$$

The expression for the kernel $\mathcal{J}$ is

$$
\mathcal{J}_{\mu \nu}=\frac{2}{1-S^{4}}\left[s_{\mu} s_{\nu}\left(2 C_{1}+C_{2}\right)-\left(s_{\mu} v_{\nu}+v_{\mu} s_{\nu}+j_{\mu \nu}\right)\right]
$$

where

$$
\begin{aligned}
& C_{1}=\sum_{\lambda}\left|\alpha_{\lambda}\right|^{2} c_{\lambda}, \\
& C_{2}=\sum_{\lambda \gamma}\left|\alpha_{\lambda}\right|^{2}\left|\alpha_{\gamma}\right|^{2} c_{\lambda \gamma}^{\prime},
\end{aligned}
$$

$c_{\lambda}=f\left(\theta_{\lambda}\right)\left[-1 / \mathcal{R}_{\lambda}+e^{-2 \mathcal{R}_{\lambda}}\left(1+1 / \mathcal{R}_{\lambda}\right)\right], c_{\lambda \lambda}^{\prime}=f\left(\theta_{\lambda}\right)\left[1 / \mathcal{R}_{\lambda}-e^{-2 \mathcal{R}_{\lambda}}\left(1 / \mathcal{R}_{\lambda}+11 / 8+(3 / 4) \mathcal{R}_{\lambda}+(1 / 6) \mathcal{R}_{\lambda}^{2}\right)\right], \quad v_{\mu}=$ $f\left(\theta_{\mu}\right)\left[-e^{-\mathcal{R}_{\mu}}\left(1+\mathcal{R}_{\mu}\right)\right], \quad s_{\mu}$ is given in (A\&), and $j_{\mu \mu}=f\left(\theta_{\mu}\right) j_{H}\left(\mathcal{R}_{\mu}\right)$, with $j_{H}(r)$ as given in (B5) of Ref. 34. As in Ref. 11, we make the following additional assumption regarding the off-diagonal elements of $c^{\prime}$ and $j: j_{\mu \nu}=\sqrt{j_{\mu \mu} j_{\nu \nu}}$ and $c_{\lambda \gamma}^{\prime}=\sqrt{c_{\lambda \lambda}^{\prime} c_{\gamma \gamma}^{\prime}}$.

The expression (A10) for exchange $J(\mathbf{R})$ is formally equivalent to the $J(\mathbf{R})$ obtained by Andres et al 34 under the additional approximation $\phi_{\mu}(\mathbf{r}) \sim \mathbf{e}^{\mathbf{i k} \mathbf{k}_{\mu} \cdot \mathbf{r}}$, thus $c_{\mathbf{K}}^{\mu}=\delta_{\mathbf{K}, 0}$, but with $\mathcal{J}_{\mu \nu}$ replaced by

$$
j_{\mu \nu}(\mathbf{R})=\int d^{3} \mathbf{r}_{1} d^{3} \mathbf{r}_{2} F_{\mu}^{*}\left(\mathbf{r}_{1}\right) F_{\nu}^{*}\left(\mathbf{r}_{2}-\mathbf{R}\right) \frac{e^{2}}{\epsilon\left|\mathbf{r}_{1}-\mathbf{r}_{2}\right|} F_{\mu}\left(\mathbf{r}_{1}-\mathbf{R}\right) F_{\nu}\left(\mathbf{r}_{2}\right) .
$$

This result emerges from an approximation for the exchange coupling commonly adopted in the literature, 11 . 34 in which only the exchange integral of the Coulomb electron-electron repulsion term is included. To our knowledge, no formal justification for this assignment is available for electrons bound to donor impurities via Coulomb interaction. However, results presented in Fig. 7 show that this is actually an acceptable approximation if the inter-donor separation is between 100 and $200 \AA$ in $\mathrm{Si}$, where relative changes $|\Delta J(\mathbf{R})| / J(\mathbf{R})$ are at most $20 \%$, keeping the same qualitative features and trends reported here for all strain and $\mathbf{R}$ values investigated. In fact, it is possible that the approximate expression obtained using (A14), which is positive-definite, instead of (A11), becomes more reliable than HL in the $R \rightarrow \infty$ limit, given the artifact of the HL expression for the exchange splitting in $\mathrm{H}_{2}$ molecule that predicts an unphysical negative $J$ for $R$ beyond 50 Bohr radii.44 45 
(a) Donor nuclear spin Si quantum computer

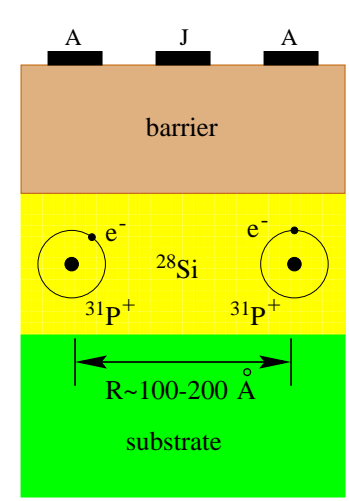

(b) Donor electron spin SiGe quantum computer

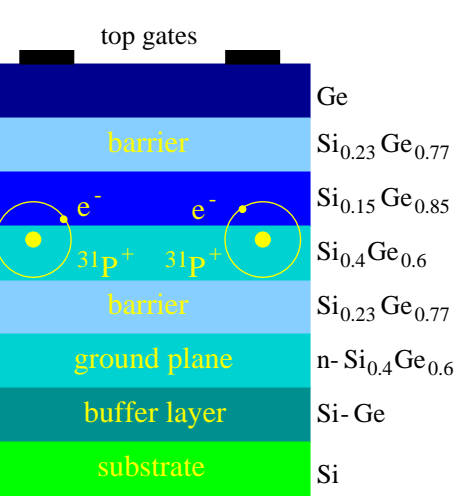

FIG. 1. (color) (a) Basic ingredients of the Silicon QC. $\mathrm{B}$ An array of substitutional ${ }^{31} \mathrm{P}$ atoms in $\mathrm{Si}$ is schematically represented in the figure by only two atoms. The active elements in the $\mathrm{QC}$ are the the electronic and nuclear spins in the ${ }^{31} \mathrm{P}$ donors. The nuclear spins $(I=1 / 2)$ are the qubits. The electrons mediate an effective exchange interaction between nuclear spins and also participate in qubit initialization and readout operations. All operations are controlled by the A-gates, placed above each donor, and by the J-gates, placed between neighboring donor pairs. A barrier layer separates the host Si crystal from the metallic gates. The A-gate is biased to bring the corresponding nuclear spin in and out of resonance with an external rf magnetic field, allowing one-qubit logic operations to be performed. Donor electrons mediate the nuclear spin interactions through their exchange coupling. Electron exchange and consequently nuclear spin interactions are turned on or $\mathrm{pff}$ by the J-gate. A uniform magnetic field $\sim 1-2$ Tesla is applied along the $z$ direction. (b) Basic ingredients of the Si/Ge QC. 4 An array of substitutional ${ }^{31} \mathrm{P}$ atoms in an alloy layer of a $\mathrm{Si} / \mathrm{Ge}$ composition-modulated heterostructure is schematically represented in the figure by only two atoms. The qubits are the donor electron spins. Given the difference in the electronic $g$-factor in Ge $(g=1.5)$ and in $\mathrm{Si}(g=2.0)$, electron-spin resonance may be controlled by the top gate, which drives the corresponding donor electron into regions of different alloy compositions. Two-qubit exchange interactions are turned on through the top gates by drawing neighboring donor electrons into a layer of small effective mass and larger wavefunction overlap.

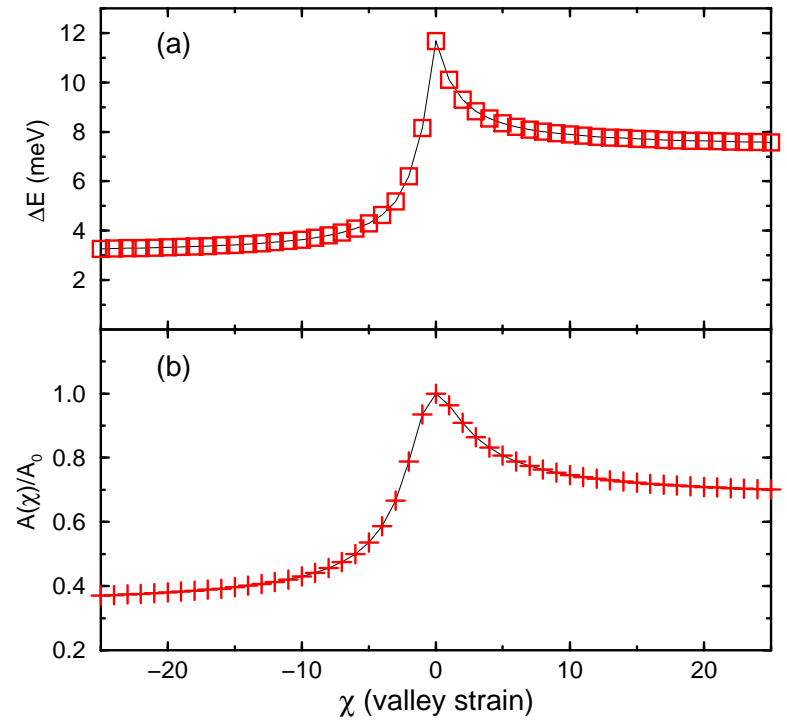

FIG. 2. (a) Energy splitting between the ground and first excited state of a single electron bound to a single ${ }^{31} \mathrm{P}$ donor in strained Si host as a function of the valley strain $\chi$. For Si commensurately grown on $\mathrm{Si}_{0.8} \mathrm{Ge}_{0.2}$, with $\chi \approx-20$, this energy splitting is about $3.3 \mathrm{meV}$, down from $\sim 12 \mathrm{meV}$ in relaxed bulk Si. For $\chi>0$, the first excited state is a doublet. At large $\chi$, this energy splitting is about $7.5 \mathrm{meV}$. (b)Hyperfine coupling of the ground single donor electron state, normalized to the unstrained host value, as a function of the valley strain $\chi$. At $\chi=-20$, the ground state hyperfine coupling is about $38 \%$ of the ground state in relaxed bulk Si. 


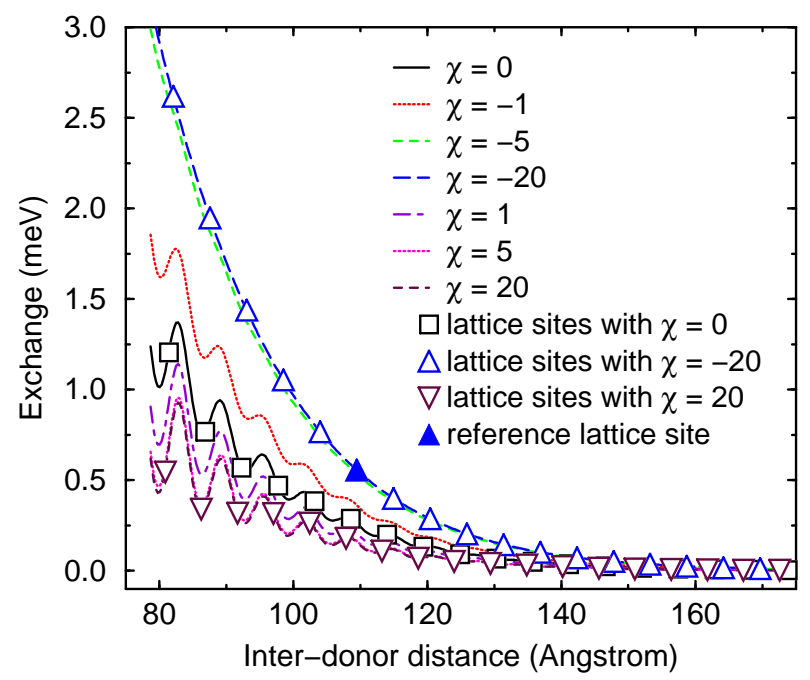

FIG. 3. Calculated donor electron exchange in $\mathrm{Si}$ as a function of inter-donor distance and valley strain $\chi$, with the two donors located along the [100] axis (with the same $y$ and $z$ coordinates). The symbols represent exchange values when both donors are on substitutional lattice sites, while the curves give results for continuously varied inter-donor distance. The filled symbol in particular refers to the configuration in which one donor is at the origin while the second donor is 20 lattice spacings away along $x$ axis. This configuration will be used as a starting reference point for the next two figures. Notice that for systems with different values of $\chi$, the symbols representing lattice sites are slightly displaced horizontally relative to each other. The reason is self-evident, as the strain is produced by growing $\mathrm{Si}$ commensurately on substrates ranging from $\mathrm{Si} / \mathrm{Ge}(\chi<0)$ to Si/C $(\chi>0)$ alloys, with varying alloy compositions that lead to different lattice spacings.

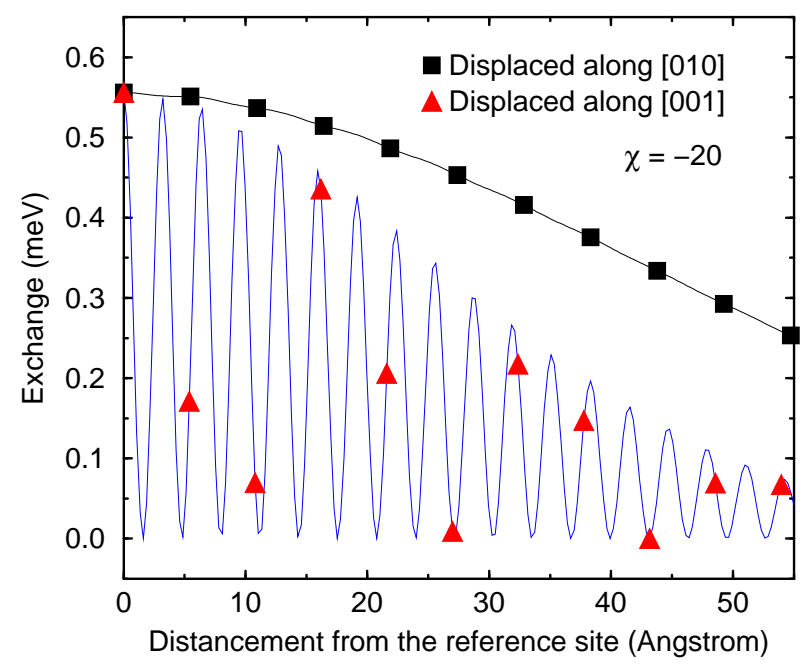

FIG. 4. Calculated exchange coupling as a function of displacements of one of the donors relative to the reference configuration in Si lattice uniaxially strained along $z$ axis with $\chi=-20$. The origin represents the situation when the two donors are in the reference configuration defined in Fig. 3. The two curves in the figure refer to the cases when one of the donors is displaced from its reference site along the $y$ and $z$ axis, respectively. The symbols represent the substitutional lattice sites along the two axis. 


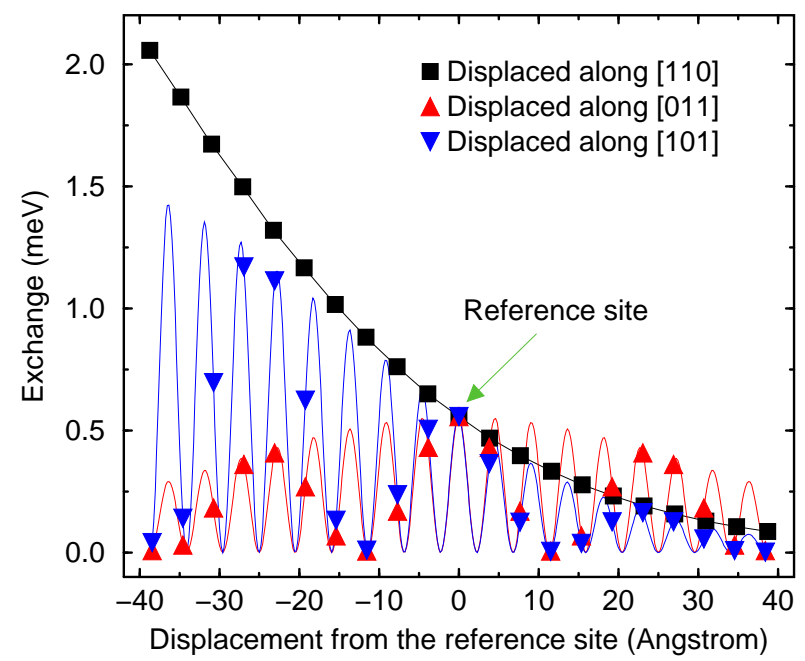

FIG. 5. Calculated exchange coupling as a function of displacements of one of the donors relative to the reference configuration in Si lattice uniaxially strained along $z$ axis with $\chi=-20$. The origin represents the situation when the two donors are in the reference configuration defined in Fig. 3. The three curves in the figure refer to the cases when one of the donors is displaced from its reference site along the [110], [011], and [101] axis, respectively. The symbols represent the substitutional lattice sites along the three axis. The curve for the [011] axis is symmetric around the reference site because the corresponding displacement along the [011] axis causes symmetric variations in the inter-donor distance $|\mathbf{R}|$.

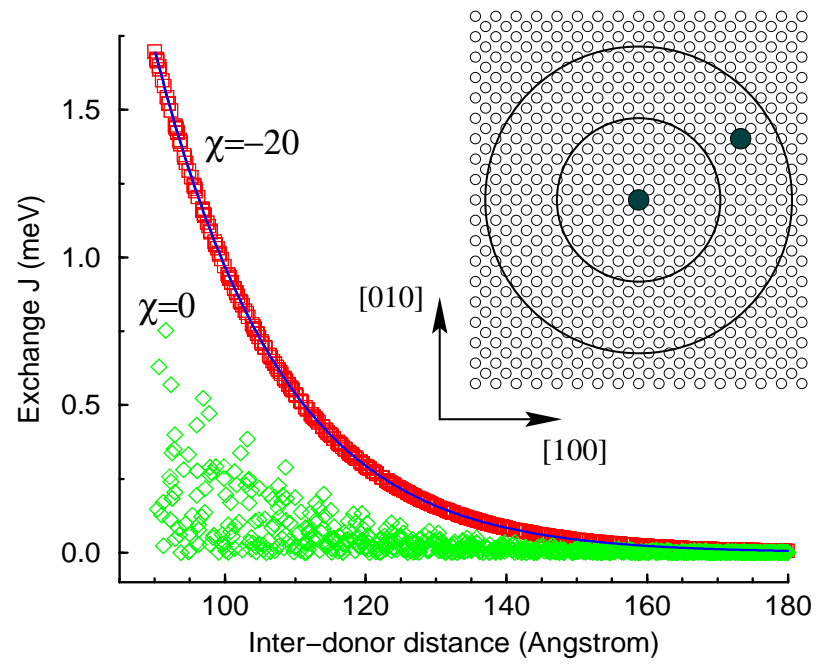

FIG. 6. Calculated exchange coupling for in-plane displacements of the donors in the $x-y$ plane for relaxed Si $(\chi=0$, diamond symbols) and strained $\mathrm{Si}$ (uniaxial strain along the $z$ direction. $\chi=-20$, square symbols). The inset, in which the filled dots represent the donors, describes schematically the positions considered for the displaced donor, which consist of all possible lattice sites between two concentric circles of radii $90 \AA$ and $180 \AA$ with the other donor positioned at the center of the circles. The data points correspond to the exchange calculated at all relative positions considered. The solid line is $J(\mathbf{R})$ for $\mathbf{R}$ along the [100] direction for $\chi=-20$. 


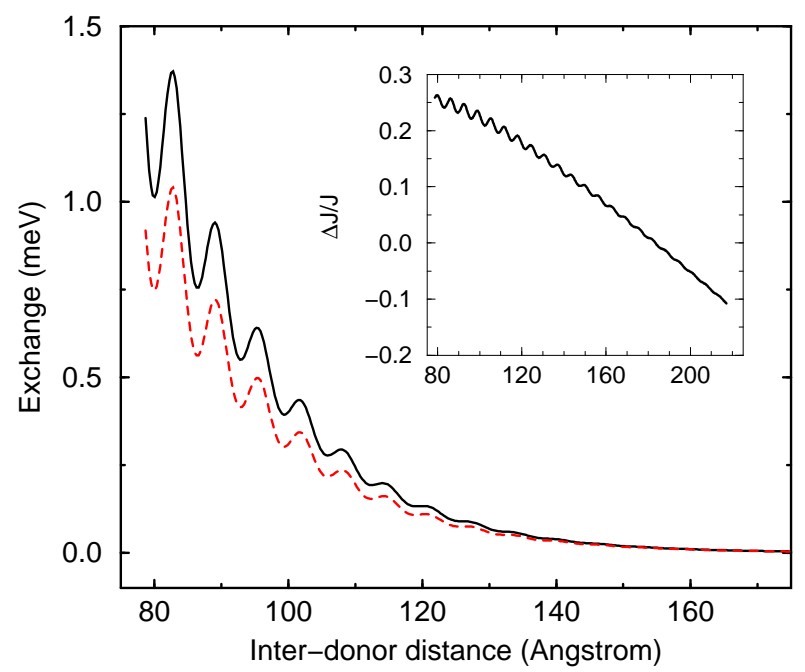

FIG. 7. Comparison of the results from calculations with the Heitler-London approximation (solid curve) and its approximate form (dashed curve) for the donor exchange in bulk relaxed Si. Without loss of generality for the comparison, the two donors here are placed along [100] axis. The inset gives the difference between these two approaches in units of the HL value for exchange as a function of the inter-donor distance. At about $180 \AA$ donor separation there is a sign change in the exchange difference $\Delta J$, while in the range of distances of practical interest, from 100 to $200 \AA$, the relative difference remains small.

${ }^{1}$ A. Steane, Rep. Prog. Phys. 61, 117 (1998).

${ }^{2}$ Special QC issue of Fortschr. Phys. 48 (2000).

${ }^{3}$ B.E. Kane, Nature 393, 133 (1998).

${ }^{4}$ R. Vrijen et al., Phys. Rev. A 62, 012306 (2000).

${ }^{5}$ B.E. Kane, Fortschr. Phys. 48, 1023 (2000).

${ }^{6}$ G. Feher, Phys. Rev. 114, 1219 (1959).

${ }^{7}$ D. Loss and D.P. DiVincenzo, Phys. Rev. A 57, 120 (1998).

${ }^{8}$ D. Mozyrsky, V. Privman, and M. L. Glasser, Phys. Rev. Lett. 86, 5112 (2001).

9 J. Levy, Phys. Rev. A 64, 052306 (2001).

10 J.L. O'Brien, et al., Phys. Rev. B 64, 161401 (2001); R.G. Clark, private communication.

${ }^{11}$ B. Koiller, X. Hu, and S. Das Sarma, cond-mat/0106259. To appear in Phys. Rev. Lett.

${ }^{12}$ K.L. Wang, S.G. Thomas, and M.O. Tanner, J. Mater. Sci. 6, 311 (1995).

${ }^{13}$ C.G. Van de Walle and R.M. Martin, Phys. Rev. B 34, 5621 (1986), and references therein.

${ }^{14}$ K. Ismail et al., Appl. Phys. Lett. 66, 1077 (1997).

${ }^{15}$ S.B. Samavedam et al., J. Vac. Sci. Technol. B 17, 1424 (1999).

${ }^{16}$ W. Windl, O.F. Sankey and J. Menéndez, Phys. Rev. B 57, 2431 (1998).

17 O. Madelung, Semiconductors-Basic Data (Springer, Berlin, 1996).

${ }^{18}$ C. Herring and E. Vogt, Phys. Rev. 101, 944 (1956).

${ }^{19}$ W. Kohn, in Solid State Physics 5, ed. by F. Seitz and D. Turnbull (Academic Press, New York, 1957).

${ }^{20}$ A. Baldereschi, Phys. Rev. B 1, 4673 (1970).

${ }^{21}$ S.T. Pantelides and C.T. Sah, Phys. Rev. B 10, 621 (1974); ibid. 10, 638 (1974).

${ }^{22}$ H.G. Grimmeiss, E. Janzén, and K. Larsson, Phys. Rev. B25, 2627 (1982), and references therein.

${ }^{23}$ D.K. Wilson and G. Feher, Phys. Rev. 124, 1068 (1961). One should note that in Fig. 1 of this reference $\delta$ was taken to be positive, leading to an inversion in the order of the doublet and triplet states for $\chi=0$, consequently affecting the ordering and degeneracy of the first excited state for all $\chi$.

${ }^{24}$ I. Balslev, Phys. Rev. 143, 636 (1966).

${ }^{25}$ L.D. Laude, F.H. Pollak, and M. Cardona, Phys. Rev. B 3, 2623 (1971).

${ }^{26}$ G. Bastard, Phys. Rev. B 24, 4714 (1981). 
${ }^{27}$ L.J. Sham and M. Nakayama, Phys. Rev. B 20, 734 (1979).

${ }^{28}$ S.J. Koester, K. Ismail, J.O. Chu, Semicond. Sci. Technol. 12, 384 (1997).

${ }^{29}$ J.C. Slater, Quantum Theory of Molecules and Solids, vol.1, (McGraw-Hill, New York, 1963).

30 J.P. Walter and M.L. Cohen, Phys. Bev. B 4, 1877 (1971).

${ }^{31}$ L.E. Oliveira and L.M. Falicov, Phys. Rev. B 33, 6990 (1986).

${ }^{32}$ J. G. Menchero, R. B. Capaz, B. Koiller and H. Chacham, Phys. Rev. B 59, 2722 (1999).

${ }^{33}$ C. Herring, in Magnetism, vol.4, ed. by G.T. Rado and H. Suhl (Academic Press, New York, 1966).

${ }^{34}$ K. Andres, R.N. Bhatt, P. Goalwin, T.M. Rice, and R.E. Walstedt, Phys. Rev. B 24, 244 (1981).

${ }^{35}$ G. Burkard, D. Loss, and D.P. DiVincenzo, Phys. Rev. B 59, 2070 (1999).

${ }^{36}$ R. de Sousa, X. Hu, and S. Das Sarma, Phys. Rev. A 64, 042307 (2001).

${ }^{37}$ P.A. Maksym and T. Chakraborty, Phys. Rev. Lett. 65, 108 (1990).

${ }^{38}$ X. Hu and S. Das Sarma, Phys. Rev. A 61, 062301 (2000); ibid. 64, 042312 (2001).

${ }^{39}$ V.M. Pudalov, A. Punnoose, G. Brunthaler, A. Prinz, and G. Bauer, cond-mat/0104347.

${ }^{40}$ G. Grosso, G. Pastori Parravicini and C. Piermarocchi, Phys. Rev. B 54, 16393 (1996).

${ }^{41}$ O. Dehaese, X. Wallart, and F. Mollot, Appl. Phys. Lett. 66, 52 (1995).

${ }^{42}$ L.I. Schiff Quantum Mechanics, Third Edition, (McGraw-Hill, New York, 1968), Chapter 8.

${ }^{43}$ J.R. Oppenheimer, Phys. Rev. 13, 66 (1928).

${ }^{44}$ C. Herring and M. Flicker, Phys. Rev. 134, A362 (1964).

${ }^{45}$ D.E. Angelescu and R.N. Bhatt, cond-mat/0012279 (2000). 